\title{
L'ALTERNANCE BINAIRE DANS LE VERS FRANÇAIS ET L'OREILLE GERMANIQUE 1 ).
}

Le vers français connaît-il ou bien a-t-il connu le mouvement soi-disant iambique, c'est-à-dire l'alternance régulière d'accent faible et d'accent fort revenant de deux en deux syllabes et que les métriciens modernes appellent rythme à alternance binaire ou plus simplement rythme alternant?

On sait que quelques théoriciens de race germanique ont cru que le vers français, ne tenant pas compte de l'accentuation naturelie, place un accent sur toutes les syllabes paires ou impaires:

Oui je viens dans son temple adorer l'Éternel 2):

Cette théorie trouve son explication psychologique dans les expériences phonétiques qui démontrent l'alternance dans une série de syllabes déclamées du type apapapa 3). Mais n'oublions pas qu'il s'agit alors de sons vides de sens et qu'il serait dangereux d'appliquer le même système d'accentuation aux combinaisons délicates et variées des mots français.

Toutefois nos métriciens de France ont attesté une tendance au rythme iambique dans quelques cas spéciaux. M. Landry, vérifiant les alternances dénuées de grâce de $M$. Saran, a cru percevoir dans la déclamation d'un artiste au Théâtre-Français deux ou trois cas de rythme alternant:

La volonté du Ciel soit faite en toute chose...

Mais l'intérêt du prince est mon premier devoir . . 4)

Et encore:

Voilà, je vous l'avoue, un abominable homme!

Évidemment 1 l s'agit dans ces trois vers d'une légère ondulation. Un Français ne saurait jamais dire le dernier vers de la façon suivante:

Voilà, je vous l'avoue, un abominable homme!

Le second hémistiche serait particulièrement lourd et disgracieux.

Un autre cas d'alternance binaire a été constaté par $M$. Landry dans la quatrième strophe de la Prière, de Sully Prudhomme, dite par une jeune Auvergnate illettrée:

\section{.... Surtout si vous saviez comment, \\ Vous rentreriez (sic) peut-être mêm[e] Tout simplement ${ }^{5}$ ).}

Dans ces exemples $M$. Landry a remarqué „la tendance d'un léger rythme périodique, et particulièrement iambique, à s'introduire dans tout groupe tant soit peu long, entre deux accents emphatiques, surtout par l'effet de la contagion, surtout s'il trouve quelque prétexte, ou du moins s'il n'est pas contrarié par l'accent du mot." Dans la déclamation pathétique $M$. Landry

1). Extrait du second chapitre d'une étude intitulée L'alternance binaire chez Jan van der Noot. (Cf. De Nieuwe Taalgids, IX, p. 1 et p. 123).

ஓ) Cf. E. Landry, La théorie du rythme et le rythme du français déclamé, Paris, 1911, p. 128.

3) O. Lote, L'alexandrin frangais, Paris, 1913, p. 273.

4) Landry, op. cit., p. 252.

s) Landry, op. cit., p. 375. 
a quelquefois cru percevoir "des répétitions de rythme, par exemple iambique, analogues à celles qui se manifestent dans d'autres mouvements par l'effet des grandes émotions, et qui parfois ne sont guère justifiées par l'accentuation normale des mots." Et il ajoute: „c'est précisément sur cette répétition spontanée de la marche de l'énergie que s'est fondée à plusieurs moments de l'histoire la convention grossière de l'alternance dans la versification 1).

Un métricien éminent, $M$. G. Lote, dont nous avons déjà cité l'étude remarquable sur l'alexandrin français, publiée en 1913, critique avec impartialité, mais non moins sévèrement les théories de $M M$. Saran et Wulff ${ }^{2}$ ). Guidé par ses tableaux généraux, où sont consignés les résultats d'une enquête qui porte sur 1400 vers environ, et admettant toutes les circonstances qui seraient favorables à la théorie alternante, il aboutit aux chiffres tout à fait probants que nous transcrivons sans commentaire:

Accent dynamique. Sur 550 alexandrins 77 hémistiches à schéma iambique ou trochaïque, soit . . . . . . . . . . . . $7 \%$.

Crêtes d'acaité. Sur 1400 alexandrins 100 hémistiches, soit . . . $3 \%$.

Durée. Sur 1400 alexandrins 7 sont d'un bout à l'autre alternants; 205 hémistiches ont l'alternance binaire, soit . . . . . . . . 7,5\%.

M. Lote conclut: $n$... Aucun Français ne considère l'accentuation iambique ou trochaïque comme un rythme essentiel, préexistant au vers, le brisant pour se le soumettre sans tenir compte de la valeur significative des mots. Parmi nos sujets, dont l'un est illettré, dont deux autres sont des acteurs, dont quelques-uns ont fait des vers, tandis que le plus grand nombre représente la masse cultivée de la nation, aucun n'a pensé à faire régner dans sa déclamation le système alternant."

Nous avons jugé utile et prudent de citer in extenso l'opinion de MM. Landry et Lote, qui par leur naissance et leurs études sont à même de se prononcer avec compétence sur un balancement léger, presque insensible qui. se répartit dans quelques circonstances favorables sur tout le vers. Le sens règle le rythme: voilà le principe dont partent les métriciens modernes. Un système préconçu, détruisant l'accentuation naturelle, peut attirer les esprits systématiques, mais ne trompera jamais le bon sens et la vérité.

Nous ne voulons pas dire qu'on ne trouve en dehors de la France de bonnes, d'excellentes études même sur le rythme du vers français! Il n'existe, pour les Hollandais, pas de meilleure introduction aux problèmes compliqués que rencontre tout étranger qui s'occupe de l'accentuation délicate des vers français, pas de guide plus sûr et en même temps plus objectif que l'article remarquable Fransche Verzen ${ }^{3}$ ), où M. A.-G. van Hamel montre qu'il excellait dans l'art d'analyser subtilement les rythmes étrangers à l'oreille germanique. Ainsi, M. van Hamel indique avec une grande justesse pourquoi les vers français s'opposent à l'alternance rigoureuse. Puisque l'accent frappe

l) op. cit., pp. 151 et 228.

9) op. cit., pp. 467-470. Cf. Saran, Der Rhythmus des französischen Verses, Halle, 1904;

Fr. Wulff, La rythmicité de Palexandrin français, Lund, 1900.

3) Het letterkundig leven van Frankrijk, vierde serie, p. 14. 
en français la dernière syllabe accentuée, l'alternance binaire serait exclue pour tous les mots de plus de deux syllabes, car l'accent secondaire dans ces mots-là n'aura jamais la même force rythmique que l'accent principal. L'erreur des métriciens germaniques accentuant par exemple:

Enfants impétueux de mon ressentiment, en appuyant trop sur pé, mon et sent a été la cause principale de leur théorie.

Nous reviendrons plus loin sur cette question assez embrouillée.

Passons maintenant aux poètes du XVIe siècle en France, et demandonsnous, comme nous l'avons fait pour ceux de notre époque, s'ils ont introduit l'alternance binaire dans leurs vers, non pas fortuitement, comme effet expressif, mais d'une façon systématique, d'après la conception de $M$. Saran. Il se pourrait que la déclamation ait changé depuis trois siècles et que les poètes de la Pléiade se soient complu à une diction artificielle détruisant l'accentuation naturelle de la langue. Voilà une hypothèse peu séduisante, qui pourtant a trouvé à mainte reprise des défenseurs et qu'il nous faut par conséquent examiner de plus près. Les Français eux-mêmes, inutile presque de le répéter, n'ont guère tenu compte des théories fantaisistes qui fleurissent si abondamment à l'étranger. Une enquête sur le vers déclamé du XVle siècle, menée par un phonéticien de naissance française, nous manque. Espérons que $M$. Lote consacrera son attention au problème de l'alexandrin binaire dans son ouvrage annoncé 1 ).

Un de ceux qui croient à l'alternance dans le vers français du XVIe siècle est $M$. Saran, mais la défense de ses idées doit être nécessairement faible, dans l'aperçu historique du livre cité ${ }^{2}$ ), párce que le problème du rythme et de la construction intérieure du vers était à peu près inconnu des théoriciens de la Plêiade. Les Arts Poétiques de cette époque ne lui fourniśsent que quelques arguments négatifs en faveur de sa théorie, qui ont déjà été réfutés par des juges compétents, et auxquels il est donc inutile de revenir ${ }^{3}$ ).

Et pourtant les écrits des théoriciens, auxquels nous ajoutons les cuvres des grammairiens et les Préfaces des recueils poétiques contiennent des renseignements précieux qui nous permettent de nier avec force l'existence d'un rythme basé sur des pieds uniformes. Voici quelque témoignages qui n'ont pas dû échapper à l'attention de $M$. Saran :

Sebillet, Art Poétique Françoys, 1548, p. 34 4):

„Pourtant voions maintenant quéles mesures et quélz nombres de syllabes reçoit le carme françois et comment il lés varie. Or sont icy les François beaucoup soulagéz au régard dés Grecz et Latins. Car ilz ne sont point astrains a certain nombre de piedz, ne a reglée aspace de temps longs ou briefz aux syllabes, comme sont les Grecz et Latins, ains seulement mesurent leurs carmes par nombre de syllabes selon le plus ou le moins ainsy que la nature du vers le requiert ${ }^{5}$ )."

1) G. Lote, La Déclamation, la Poétique et la Versification depuis le XVIe siècle.

2) op. cit., p. 12.

3) Voir notamment van Hamel, Museum, 1906 et Vossler, Archiv, 1906.

4) Ed. Gaiffe, Paris, 1910.

5) Nous devons cette citation intéressante à l'amabilité de M. J.-J. Salverda de Orave. 
Et voici une citation non moins importante, empruntée aux Recherches de la France 1), d'Estienne Pasquier, Tome I, liv. VII, p. 710:

"Leurs vers (c.-à-d. ceux des Grecs et Latins), si ainsi me permettez de le dire, marchent 8 vont avec leurs pieds, les nostres glissent $\&$ coulent doucement sans pieds, voire quand bien il n'y aurait point de rime en laquelle toutesfois gist l'accomplissement de nos vers. Chose que Ronsard nous voulut, representer par cette Ode, qui est la quinzième du troisiesme livre des Odes, sur la naissance de François, fils du Roy Henry deuziesme

En quel bois le plus séparé etc. ${ }^{2}$ )

Je vous laisse le demeurant, pour vous dire que cette Ode contient une longue texture $\&$ trainée de vers qui n'ont point de pieds, comme les Grecs \& Romains, \& sont pareillement sans rimes, es quelles gist la principale grace des nostres. Ce neantmoins vous les voyez nous succer l'aureille par leur douceur, antant \& plus que tous les Exametres \& Pentametres des autres, desquels pour cette cause il ne faut mandier les vers mesurez: car de combien se rend nostre Poësie plus douce, quand elle est accomplie de la rime, en laquelle, comme j'ay dit, reside sa principale beauté?"

Une citation analogue se trouve parmi les lettres de P.-C. Hooft. D'après lui, un des plus grands poètes français („een onder hunluyden die sich de Dichtkunst vermeten heeft boven andere te verstaen, ende van de meeste daer voor gehouden is geweest) aurait dit:

"La poësie des Grecs et Romains mesure ses vers par certains nombres de pieds, composez tant de longues que brefves syllabes sans rime; nous au contraire faisons entrer dedans nos vers toutes sortes de syllabes, soyent longues ou brefves, sans aucun triage; ains suffit qu'ils aboutissent en parolles de pareille terminaison ${ }^{3}$ ).

Et pourquoi cette distinction entre vers latins et vers français nous paraîtelle si remarquable? Parce que, si les poètes avaient connu le rythme alternant; ils l'auraient facilement mis en rapport avec le iambe et le trochée latins. Long $(-)$ et bref $(-)$, principes d'une versification quantitative, auraient été appliqués au vers accentuel, comme le font les traités de prosodie des langues germaniques.

Sous ce rapport les essais de vers métriques qui n'ont jamais eu un éclatant succès en France sont instructifs. Le problème aurait été plus facile à résoudre si l'accent frappait régulièrement toute deuxième ou troisième syllabe. Un de ceux qui firent une tentative de vers métriques fut Jacques de la Taille, qui en 1573 écrivit La Maniere de faire des vers en franfois, comme en Grec $\mathcal{E}$ en Latin ${ }^{4}$ ). Il conseille de se servir de pieds simples, iambe, trochée, spondée et dactyle, mais non pas exclusivement du iambe et du trochée, ce qu'il aurait certainement fait si le vers français du XVIe

1) Amsterdam, Trévoux, 1723.

2) Nous supprimons les quatorze vers blancs qui suivent.

5) P. C. Hooft's Brieven, publ. p. J. van Vloten, I, p. 438. Nous n'avons pu verifier l'exactitude de cette citation parce que nous avons cherché en vain le nom du poète français dont il s'agit.

4) Cf. T. Rucktäschel, Einige arts poétiques aus der Zeit Ronsards und Malherbes, Leipzig, Gustav Fock, 1889, p. 26. 
siècle avait connu l'alternance binaire. Les pieds - et $-\smile$ se seraient alors le mieux adaptés à l'accentuation française 1 ).

Et puis il y a encore ceci: les défenseurs les plus acharnés du rythme binaire ont dû reconnaître qu'un grand nombre de vers français étaient rebelles au principe établi en dehors des textes, et auquel ils font ensuite entrer de gré ou de force les exceptions multiples. Tel par exemple Harsdörffer, „corrigeant" quelques vers de Ronsard:

Cơntrē (au lieu de cōntrě) lě māl d'ămōur

quě tōus lěs mâtx ěxcēdĕ

L'ărtīfícē (au lieu de L'ārtifīicĕ) n'innvēntě

ŭn plūs prěsēnt rěmēdě etc. 2$)$.

Tels encore Gottsched, W. Heinse et J-G. Gruber en Allemagne ${ }^{3}$ ), et chez nous Constantijn Huygens 4).

S'il est donc peu probable, d'après tout ce qui précède, que le vers français, non seulement celui de notre époque mais encore celui du XVIe siècle obéît à l'alternance binaire telle que certains métriciens de race germanique l'ont comprise, il faut rejeter toute théorie qui explique la naissance du rythme nouveau chez les poètes de la Renaissance flamande et néerlanlandaise, chez Jan van der Noot et ses contemporains par la simple imitation de la poésie française 5 ).

Comment faudrait-il s'expliquer du reste cette influence directe? Il est certain que les étrangers se font fréquemment une idée fausse du rythme français qu'ils remplacent par un rythme subjectif, illusoire. La poésie qui n'est pas nôtre, et que nous ne saisissons par conséquent qu'après un effort intellectuel plus ou moins grand, nous parvient surtout par la lecture, plus rarement par la déclamation, et même dans le dernier cas l'oreille peut nous duper étrangement - nous pensons à $M$. Saran croyant constater au ThéâtreFrançais plusieurs alternances dont $M$. Landry a démontré la nullité. Après notre enquête précédente qui nous a conduit à une réponse négative, nous avons le droit de nous poser une question d'intérêt plus général, celle de savoir comment les métriciens néerlandais, à partir du XVIe siècle, ont pu croire a l'alternance binaire dans le rythme du vers français. Cette erreur, à notre avis, est due à deux causes: $a$. La cause secondaire doit être cherchée dans le vers français lui-même; $b$. La cause principale se trouve dans la structure du vers néerlandais.

Nous nous proposons de développer le premier point et d'indiquer brièvement l'intérêt $\mathrm{du}$ second, l'étude du vers néerlandais méritant d'amples développements qui déborderaient le cadre de cet article.

Nous avons va plus haut que les phonéticiens français ont constaté quel-

1) Cf. Tobler, Vom Franz. Versbau alter und neuer Zeit, Leipzig, 1894, p. 5.

2) Saran, op. cit., p. 172.

3) Saran, op. cit., pp. 174-176.

4) Voir plus loin.

5) Nous jugeons inutile de discuter la page que M. Saran consacre à la poésie néerlandaise. Six vers du Lofsanck -van Bacchus, de Daniël Heinsius - et quels vers! - ne sauraient prouver la justesse de son principe. (Cf. op. cit., p. 187). 
ques rares cas d'alternance quasi-régulière. Pour bien comprendre de quelle façon ce type de vers iambique a propagé une accentuation fausse parmi les étrangers, nous résumons les idées exprimées par Constantijn Huygens, le poète de Costelick Mal, de Zeestraet et des Sneldichten dans sa correspondance avec P.-C. Hooft, de 1623 1), et dans sa controverse avec Corneille, de 1663 2).

Huygens, qui se piquait de bien savoir le français, avait remarqué qu'un certain nombre de vers se prêtent assez docilement à l'alternance binaire.

Enfants impétueux de mon ressentiment

Que ma douleur séduite embrasse aveuglément

pourrait être scandé ainsi :

Enfants impétueux de mon ressentiment

Que ma douleur séduite embrasse aveuglément.

Il oublie que cette scansion introduit un nouvel accent dans impétueux, ressentiment et aveuglément et qu'elle appuie trop sur mon et ma. Mais comme il trouve à ces vers des "cadences si jolies et si naturelles", les vers qui ne répondent pas à ce schéma sont défectueux, "choquent le bon lecteur" 3). Étrange logique!

Pour ne pas être obligé de condamner la majorité des vers français suivons notre théoricien de loin sur cette pente rapide et dangereuse Huygens compte également parmi les "bons vers" celui-ci :

Oui Cinna contre moi moi-même je m'irrite

et ces deux vers de La Veuve:

Moi même je fais mon supplice...

Et forment ma crainte et mes vœux.

Va-t-il maintenant franchir tous les obstacles pour aboutir à la scansion uniforme, les oreilles bouchées, de tous les vers français? Il n'aurait certes pas été le premier - ni le dernier, hélas! - à professer cette fâcheuse doctrine. Non, il s'arrête à mi-chemin et, toujours guidé par sa cadence „si jolie et si naturelle" il découvre dans les tragédies de Corneille un certain nombre de vers qui, scandés d'après ce principe, sont mal accentués; celui-ci par exemple:

Vous prenez sur mon âme un trop puissant empire, où prenez empêche l'ondulation de faible à forte.

Au lieu de reconnaître la fausseté de son principe, Huygens condamne ce vers. Il en veut bien admettre un moment la correction par un déplacement d'accent: Vous prenez etc., "mais, conclut-il, ce n'est plus le même vers, parce que ce n'est plus le premier mouvement, et ce premier mouvement est faux parce que la nature de l'accent y répugne." Ainsi notre poète se débattit en vain dans un cercle vicieux d'où Corneille, ne comprenant pro-

1) P. C. Hooft's Brieven, publ. par J. van Vloten, I, pp. 434-449. On trouve un résumé de cette discussion dans Opmerkingen over Nederlandse versbouw, de M. C.-G.-N. de Vooys, Taal en Letteren, 1906, fasc. 4, 5, p. 181.

2) M. A.-G. van Hamel a consacré une étude intéressante à ce sujet dans la Zeitschrift für franz. Sprache und Litteratur, XII, 4, 6, p. 191 et dans Het letterkundig leven van Frankrijk, IV, p. 3. En 1905 il est revenu au même sujet dans une communication faite au congrès de la langue française à Liège, à propos d'un écrit plus ancien de Huygens.

3) Van Hamel, Ztschr. f. fr. Spr. u. Litt., XII, 4, 6, p. 194. 
bablement rien aux raisonnements subtils de son critique, ne se donna pas la peine de le faire sortir.

Huygens s'appuie dans son raisonnement sur un argument qui mérite d'être examiné de plus près. Il soutient l'utilité d'une accentuation uniforme pour tous les vers en vue d'une déclamation chantée: "Comme tout poète chante, toute poésie devrait être bien chantable" 1). A cet effet il exige un rythme qui soit identique pour tous les vers du couplet 2 ). Déjà une quarantaine d'années plus tôt - en 1623 - il avait développé cet argument dans une dissertation sur l'uniformité des vers et la mesure des pieds ${ }^{3}$ ). La poésie, selon lui, n'est autre chose qu'une déclamation chantée, vu que "le chant consiste, au fond, à rattacher légèrement les mots l'un à l'autre en "portant" la voix" 1).

L'application de cette idée très juste pour les textes des chansons, a été moins heureuse: Huygens ne réussit pas à faire une distinction bien nette entre les vers déclamés et ceux qui sont mis en musique. Il exige en premier lieu un rythme conforme à la mélodie dans les stances italiennes, les couplets français et les vaersen hollandais, mais semble étendre son principe aux autres genres de poésie: Hooft, qui ajoute ses critiques en note à la dissertation de Huygens, répète deux ou trois fois que l'observation du dernier peut être juste pour le chant, mais que la tragédie, l'épopée et l'épître n'exigent pas de rythmes uniformes ${ }^{5}$ ). Et même dans la poésie chantée deux vers de longueur différente se plient souvent à la même mélodie. Le peuple (de hujslujden) chante, d'après Hooft, les deux vers suivants de la Chanson de Gerrit van Velsen sur les mêmes notes:

Die schand en schiê my nimmermeer

Sprack Gerrit van Velsen tot sijnen landsheer ${ }^{6}$ ).

Nous arrivons à la seconde des causes nommées plus haut de la conception erronée des métriciens néerlandais.

Les vers du type Enfants impétueux de mon ressentiment peuvent induire en erreur l'oreille germanique quant à l'accentuation d'autres vers français; ils peuvent même servir de modèle à des imitations directes dans les langues

(1) Comparez ce que dit Ronsard dans l'Abrégé de l'art poétique françois, éd. MartyLaveaux, t. VI, p. 353: "Je te veux aussi bien advertir de hautement prononcer tes vers quand tu les feras, ou plus tôt les chanter, quelque voix que puisses avoir."

2) Van Hamel, l. c., p. 195.

3 Voor de eenparicheit der dichten ende van de voetmaet, insérée dans l'édition citée des Lettres de Hooft, I, p. 434.

4) "Het gedicht is niet anders als canora (sic) dictio oft een singende uytspraek, . . . gemerckt het singen eygentlijck is de woorden dichten, dat is: met het slepende gevolgh van de stem licht aan den andren hechten."

j) Hooft constate, dans sa Granida, le rythme iambique avec trois variantes; - $\smile-\smile-$; $\smile--\smile \smile-$ et $-\smile-\smile \smile-$; il rejette donc l'alternance rigoureuse. Ses critiques sur les observations de son ami Huygens dénotent du reste des vues bien plus profondes sur la structure du vers français.

6) Nous pouvons ajouter cet exemple instructif, qui ne s'explique que par l'isochronisme des pieds rythmiques, à ceux que nous avons cités dans nos articles précédents sun l'accentuation du vers néerlandais:

Die schand en schle my nimmermeer

Sprack Gerrit van Velsen tot siljnen landsheer.

Cf. Het isochronisme in het Nederlandse vers et Hoger rythme, publiés dans De Nieuwe Taalguds, 1X, 1, 3. 
non-romanes, - leur influence restreinte ne suffit pas à expliquer le changement profond qui s'est opéré vers 1560 dans la technique des vers néerlandais, chez Jan van der Noot d'abord, chez d'autres innovateurs ensuite. Non, il faudra chercher des lois plus générales auxquelles obéissent non seulement quelques poètes isolés, mais, aux siècles suivants, plusieurs générations d'artistes.

Où trouvera-t-on ce courant irrésistible, regrettable souvent?

Si l'oreille germanique a cru entendre, avec une persistance singulière, le rythme alternant dans les vers français, on peut supposer que ce rythme lui était familier, qu'il fait partie du génie même de sa langue. Si cette hypothèse est fondée, on devra chercher dans la poésie antérieure à 1560 , dans le vers accentuel du moyen-âge, dans la chanson populaire, dans les productions non-savantes surtout, mais pas exclusivement, les germes de la nouvelle versification. Ainsi nous arrivons à ces trois questions, dont nous signalons ici toute l'importance, sans les examiner à fond: Le rythme alternant dans le vers néerlandais est-il vraiment aussi nouveau, au XVIe siècle, qu'on le croit généralement? Jusqu'à quel point ce rythme a-t-il été inconsciemment le principe du vers non-syllabique antérieur? Sous quelles influences se manifeste-t-il avec assez d'évidence pour qu'on ait pu croire à une nouveauté?

Nous espérons communiquer les résultats de cette enquête dans une étude prochaine.

Groningen.

J. VAN DER EIST.

\section{L'ESTHÉTIQUE D'ANDRÉ CHÉNIER D'APRÈS UN OUVRAGE POSTHUME 1).}

A la mémoire de Mademoiselle M. E. Loke.

Poète lyrique comme depuis Ronsard et Du Bellay le classicisme n'en avait pas produit en France, André Chénier comprit de bonne heure qu'il lui fallait, pour son propre usage, modifier les règles et préceptes de Malherbe et de Boileau dont la rigueur avait eu pour effet d'étouffer dans la poésie française précisément le véritable lyrisme 2). Autant, ou plus encore, il s'est attaqué à la sécheresse de ses contemporains, les pâles versificateurs du XVIIIle siècle, ces faiseturs d'épigrammes et de madrigaux dont Voltaire luimême n'était trop souvent que le premier. Ainsi il s'est cru obligé d'exposer, et à plusieurs reprises, ses idées sur la poésie et sur l'art.

Tout d'abord il l'a fait en artiste, lorsque, dans la fameuse Epître à Lebrun, il nous décrit à la manière de La Fontaine, sa méthode de travail. Il semble

1) L'Essai sur les Causes et les Effets de la Perfection et de la Décadence des Lettres et des Arts, publié par M. Abel Lefranc d'après les Manuscrits originaux, d'abord dans la Revue de Paris du 15 octobre et du ler novembre 1899 , puis, tout récemment, réuni avec d'autres fragments posthumes, en volume et sous le titre d'CEuvres Inédites d'André Chénier, Paris, Champion, 1914.

2) Cf. P. Glachant, André Chénier critique et critiqué, Paris, Lemerre, chap. 1II, p.90 et suiv. 